\title{
STATUS OF CHIPS: A NASA UNIVERSITY EXPLORER ASTRONOMY MISSION
}

\author{
Mr. Will Marchant, Dr. Mark Hurwitz, Dr. Michael Sholl, Dr. Ellen Riddle Taylor \\ Space Sciences Laboratory - University of California, Berkeley \\ GrizzlyPeak@Centennial \\ Berkeley, CA 94720
}

\begin{abstract}
In the age of "Faster, Better, Cheaper", NASA's Goddard Space Flight Center has been looking for a way to implement university based science missions for significantly less money. The University Explorer (UNEX) program is the result. UNEX missions are designed for rapid turnaround with fixed budgets in the \$10-\$15 million US dollar range. The CHIPS project was selected in 1998. The CHIPS mission passed the Design Verification Review in April 2001 and is now proceeding into implementation with a launch in mid-2002. Many lessons have already been learned from the CHIPS UNEX project. The 2000 paper discussed the early issues surrounding the use of commercial satellite constellations and the politics of small satellites using foreign launchers. The difficulties of finding a spacecraft in the UNEX price range were highlighted. The advantages of utilizing Internet technologies from the earliest phases of the project through communications with the spacecraft on orbit were discussed. The 2001 paper will discuss the implementation status of CHIPS, the first of this new class of NASA mission, and the lessons learned. The current state of the program will be summarized and the project's plans for the future will be charted.
\end{abstract}

\section{Introduction}

The Cosmic Hot Interstellar Plasma Spectrometer (CHIPS) is the first mission to be funded through NASA's University-Class Explorer (UNEX) program. The explicit goal of UNEX is to demonstrate that significant science and/or technology experiments can be accomplished in a limited schedule and constrained budget. UNEX requires a development time of approximately two years and a mission budget on the order of \$10$\$ 15 \mathrm{M}$ inclusive of the launch vehicle and mission operations.

The primary science objective of CHIPS is to provide spectral sky maps of the scientifically critical extreme ultraviolet (EUV) wavelength band between 90 and $260 \AA$. The CHIPS full- sky survey maps will help determine the electron temperature, ionization conditions, and cooling mechanisms of the million-degree plasma believed to fill the local interstellar bubble.

The CHIPS mission was initially proposed as part of the 'Secondary Payload Program' aboard a FAISAT commercial communications satellite. However, in late 1998, concerns arose from the Office of Science and Technology Policy (OSTP) because of the satellite's scheduled launch on a Cosmos rocket. Consequently, the CHIPS team baselined using a dedicated small satellite bus developed by SpaceDev, Inc in Poway, CA. The satellite was to be launched aboard a three-stage Delta II launch vehicle as a secondary payload with a GPS 
refurbishment mission. However, recent notification of modifications and weight increase of the GPS primary payload has made this option potentially infeasible.

Despite continued uncertainty with the launch vehicle, CHIPS recently held their Design Verification Review in April 2001 and is on track for a mid-2002 delivery.

\section{$\underline{\text { Design Overview - Instrument }}$}

The CHIPS instrument, developed at the Space Sciences Lab at UC-Berkeley (UCB), is a multi-channel spectrograph. The instrument consists of an array of entrance slits and cylindrical diffraction gratings that disperse and focus diffuse extreme ultraviolet radiation onto a photon-counting microchannel plate detector. A dedicated electronics unit provides all instrument specific functions, including power distribution and control, photon data processing and instrument safing. The full Spectrograph with electronics weighs approximately $30 \mathrm{~kg}$ and uses about $20 \mathrm{~W}$.

Figure 1 provides an illustration of the instrument. Light enters the spectrograph through six independent channels. The entrance apertures are narrow slits, covered by simple once-open covers. Light passing through the entrance slits illuminates individual, identical cylindrical diffraction gratings. The gratings disperse and focus the diffuse extreme ultraviolet radiation onto a single detector through a filter assembly. The bottom of the figure shows the instrument detector assembly, a planar, photon-counting microchannel plate system with a crossed delay line anode. In-band photon locations are determined from the anode, which converts the light into analog electronic pulses. RF amps amplify the anode signal and the pulses are converted into digitized coordinates by the Time to Digital Converter (TDC) in the CHIPS Instrument Electronics
Box (EBOX). The digitized events are then transmitted to the instrument computer (DPU) for processing. The DPU packages and formats all instrument data for transmission to the spacecraft Single Board Computer (SBC) via redundant asynchronous RS-422 links.

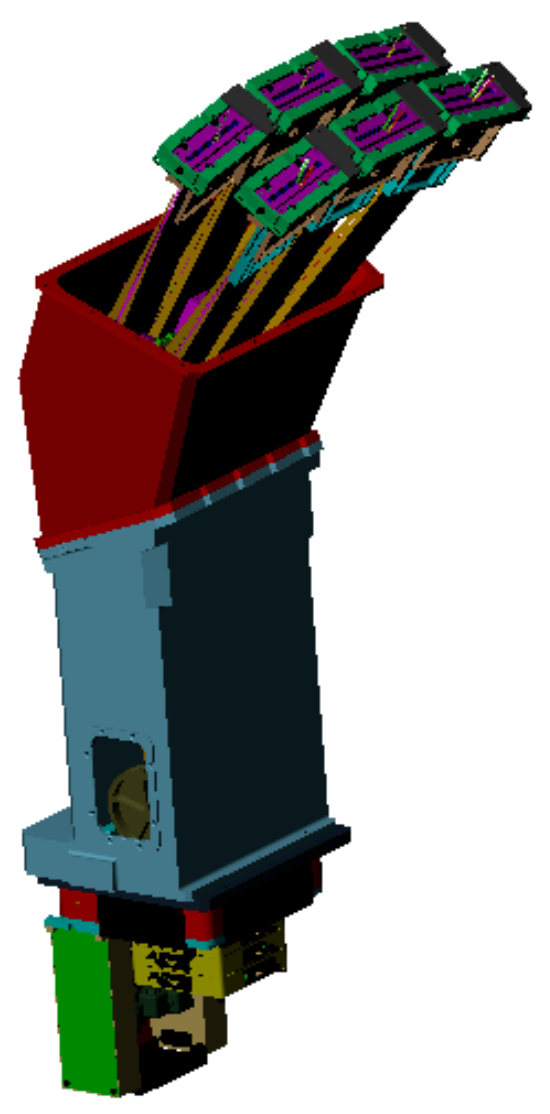

Figure 1: CHIPS Spectrograph

\section{Design Overview - Spacecraft}

The CHIPS Spacecraft (CHIPSat) is a 3-axis stabilized spacecraft using 4 momentum wheels, 3 torque coils, sun sensors, a magnetometer, and rate sensors to provide $\sim 2$ degrees attitude accuracy and control. A Moon sensor, co-aligned with the science instrument, will provide feedback for science attitude calibration. The flexible and capable configuration not only provides the ability to accomplish a full sky survey of the entire 
celestial sphere, but also allows very deep observations of regions of particular interest.

The mass for the entire satellite inclusive of the instrument is less than $70 \mathrm{~kg}$. Dualjunction $\mathrm{GaAs} / \mathrm{InP} / \mathrm{Ge}$ body-mounted solar arrays with Nickel Cadmium batteries provide the necessary $40 \mathrm{~W}$ of power. The primary solar arrays are body mounted on three sides of the spacecraft with small keep-alive arrays positioned on the other sides providing enough power to run critical subsystems regardless of the spacecraft's attitude.

Signals from the instrument and spacecraft subsystems are acquired, formatted and stored by the spacecraft's data handling system, a single-board flight computer consisting of a
Motorola Power PC 750 CPU. CHIPSat will use an S-band transceiver for the RF communications. A pair of quadrifilar antennas mounted on opposite sides of the spacecraft provides an omnidirectional beam pattern.

Scientific, instrument and spacecraft housekeeping data is stored within spacecraft memory, combined with orientation data, and downlinked several times daily to S-band ground stations at UCB and Adelaide, Australia. Data is received, archived, and monitored at the Mission Control Center (MCC) located at the SpaceDev facility in Poway and then sent to the CHIPS Science Operation Center (SOC) at UCB.

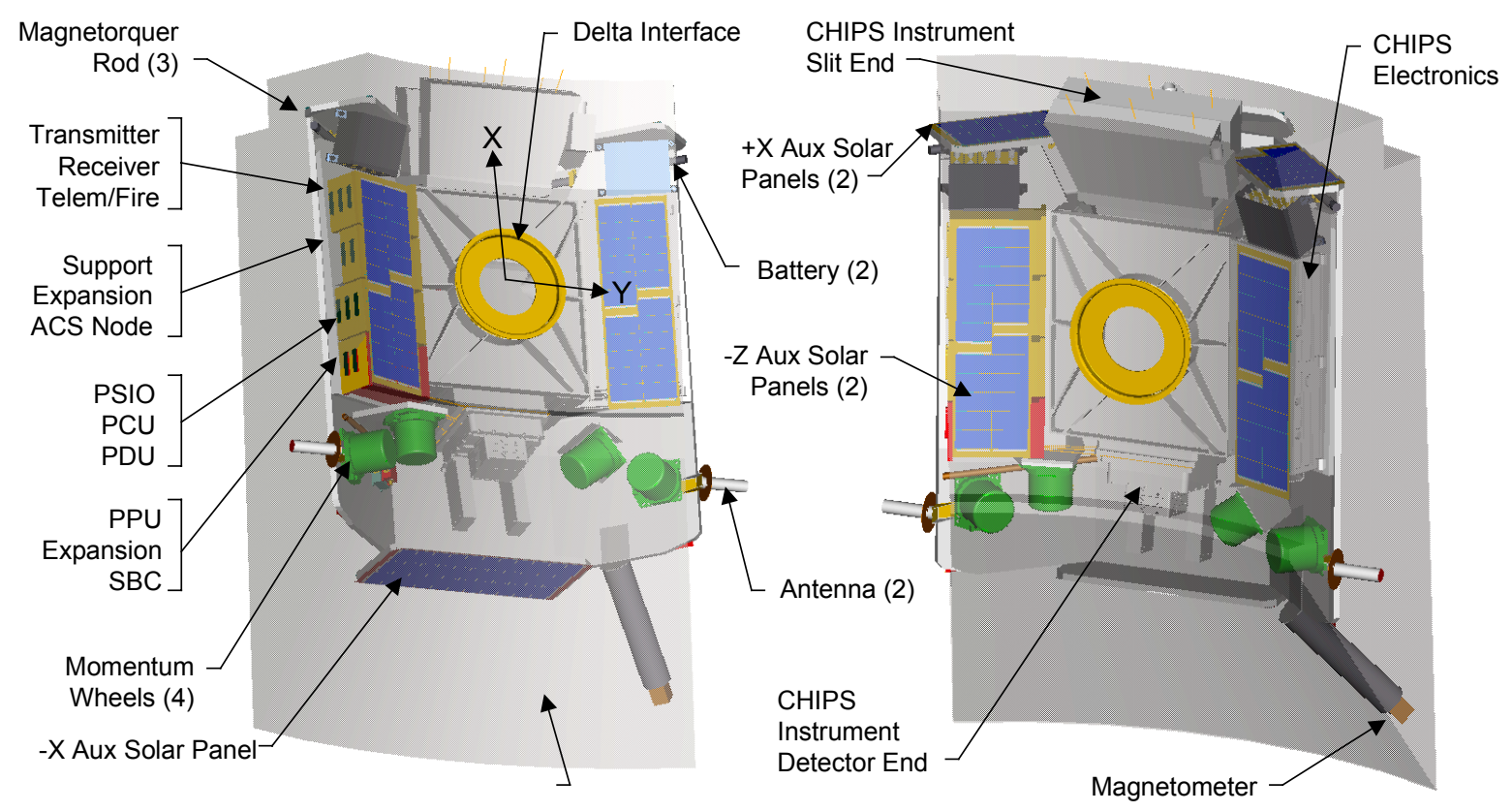

Figure 2: CHIPSat Spacecraft Bus

\section{System I\&T - Using the Internet}

In an effort to significantly lower costs and shorten the System Integration and Test (I\&T) Phase, CHIPS has teamed with the OMNI project at GSFC to demonstrate Internet protocols all the way from the scientists sitting at the Science Operations
Center (SOC) to the payload interfaces onboard the spacecraft. The TCP/IP stack and existing utilities (such as FTP) built into the WindRiver VX Works RTOS in the spacecraft single board computer provide an essentially "Free" infrastructure for moving data between the spacecraft and the ground systems. 
The CHIPS team has already used the Internet extensively to support early "virtual integration" of components and has found that it offers a huge advantage for distributed projects. The entire attitude and determination system (ADCS) is being provided by Dynacon in Canada, the Instrument hardware is at UCB in Northern California, much of the Instrument software is being developed in Virginia, and the spacecraft bus is at SpaceDev in Southern California. Using the internet, the CHIPS team has been able to connect these systems virtually and work out interface problems prior to final subsystem flight build, test and delivery. This phased development of integration and testing has proven to greatly ease the cost and schedule of bringing hardware together at specific points in the project.

Through the Internet, science data can be obtained using the facilities at the Space Science Lab at UCB (See Figure 3), virtually transferred through the spacecraft system at SpaceDev, and routed back to the Science Operation Center at UCB.

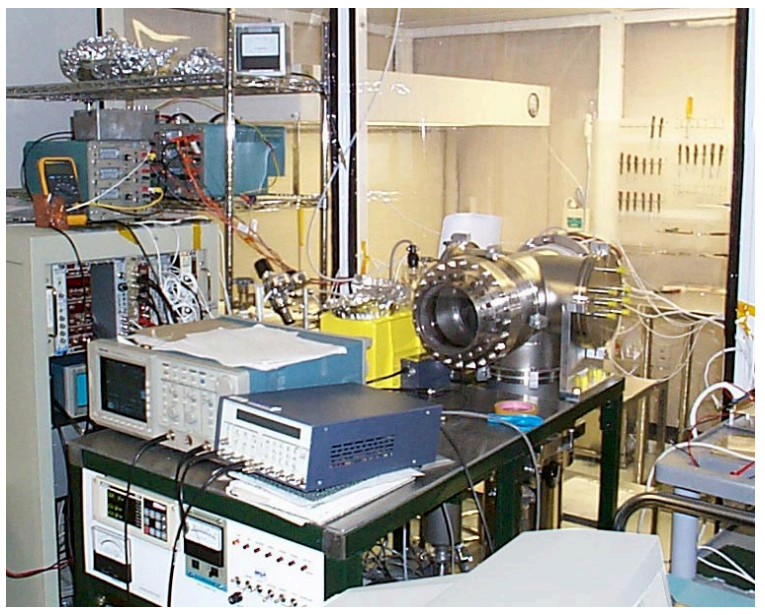

Figure 3: Detector Vacuum Chamber at UCB Facility

Figure 4 shows the set up of this "End-toEnd" testing completed with prototype, Engineering Test Unit (ETU), or flight instrument and spacecraft subsystems.

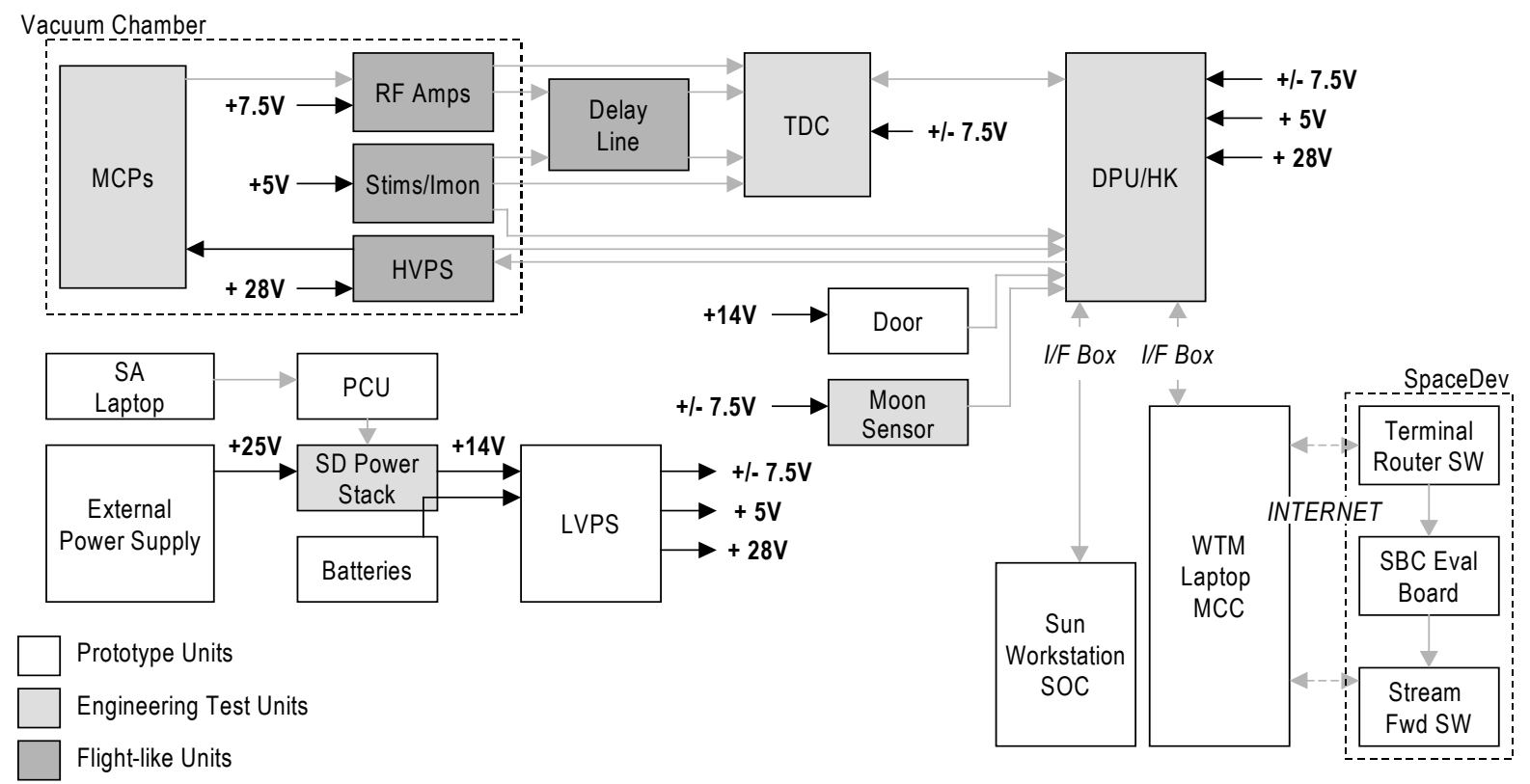

Figure 4: End-to-End Test Set-up and Interfaces 
During testing, science photon data obtained from the detector assembly (MCPs, amps and stims) is transferred to the instrument DPU/HK board for packaging and transfer to the SpaceDev system over the Internet. Virtual terminal routers are used to transfer the serial data between the DPU, the Mission Control Center (MCC) at SpaceDev in Southern California, and the Science Operations Center (SOC) at UCB in Northern California. After the science data was transferred to SpaceDev, timetags and simulated attitude information was inserted into the data stream at either their Single Board Computer (SBC) evaluation board or their prototype flight computer and then stream forwarded back over the Internet to a SOC Sun Workstation at UCB where it was archived as ASCII files.
Similar to this testing with the Instrument, SpaceDev has also been able to integrate other components of the full observatory system by using the Internet to "extend" the serial links between these subsystems (see Figure 5). All standard TCP/IP services (FTP, telnet, time protocols), as well as the custom UDP/IP socket interfaces, have been tested. At the SpaceDev Facility, the SBC is interfaced to telemetry and power control boards over a SSP serial bus, representing most of the major devices on the SSP bus. The last device on the SSP bus is the ACS node, which has been tested over the Internet using PC-based code that will eventually be ported to VXWorks.

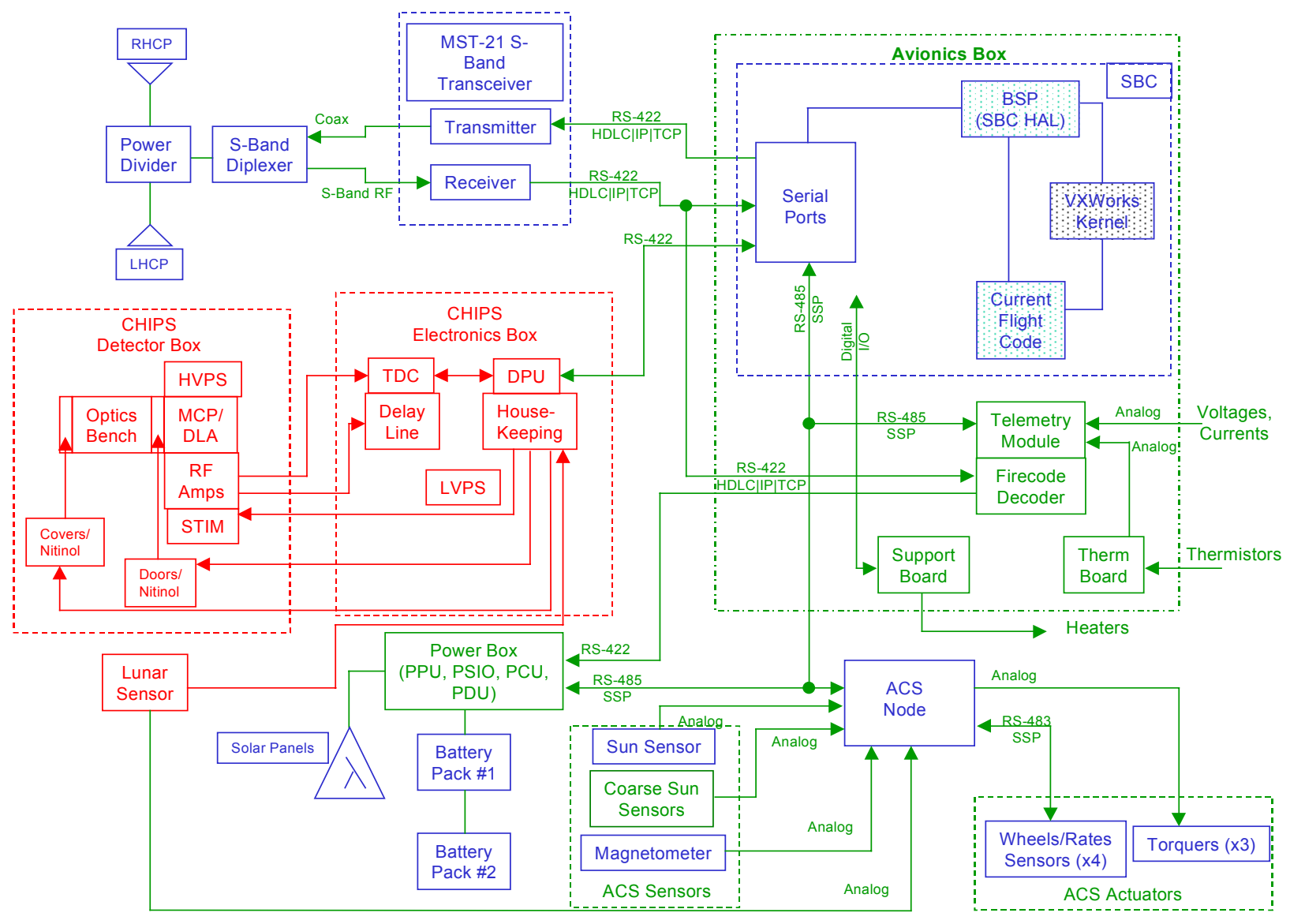

Figure 5: CHIPSat End-to-End Set-Up and Interfaces 


\section{Conclusion}

The CHIPS program is currently on-track for a mid-2002 launch. Delivery of the Instrument to SpaceDev is expected in November 2001 for integration and environmental testing prior to launch.

The CHIPS program has already benefited considerably from the use of COTS software systems. This was largely enabled by the use of the various Internet protocols and utilities all the way from the spacecraft through to the Principle Investigator's science team.

The proper use of these tools results in a number of benefits:

- Development time is reduced because specifications and tools already exist.

- Testing time is greatly reduced because the tools and protocols are well established in the worldwide user community.

- It is easy to get technical support for these tools.

- Because the Internet is part of the operational structure, much of the testing is directly applicable towards confirming the operational configuration of the end to end system.

- Early integration activities are encouraged, which helps discover specification and implementation problems early in the project when they are easier, and cheaper, to fix. 